\title{
Aetiology
}

\section{Existing psychological problems predict depression in primary care better than sociodemographic factors}

Barkow K, Maier W, Ustun TB et al. Risk factors for new depressive episodes in primary health care: an international prospective 12-month follow-up study. Psychological Medicine 2002 May;32:595-608.

\section{QUESTION: What are the risk factors for depressive episodes in primary care?}

\section{Design}

Population-based cohort study with 1 year of follow up. Outcome assessors were blinded to baseline assessments.

\section{Setting}

15 primary care centres in 14 countries in Africa, Asia, Europe, North America and South America.

\section{Participants}

Stratified sample of 2445 people without initial depression in the World Health Organisation 'Psychological Problems in General Health Care' study who attended a participating primary care clinic. All were younger than 66 years (mean age 41 years); $63 \%$ women.

\section{Assessment of risk factors}

Sociodemographic variables (age, gender, martial status, years of formal education, employment, location); psychological and psychiatric problems, and social disability measured using questionnaires at baseline.

\section{Main outcome measures}

Presence of a depressive episode at 12-month follow up measured using ICD-10 criteria. Logistic regression assessed the link between depressive episodes and risk
Source of funding: None listed.

For correspondence: K Barkow, Department of Psychiatry, University of Bonn, Germany.

\section{factors.}

\section{Main results}

After 12 months, $4.4 \%$ of participants had a new depressive episode. Factors related to the development of newdepressive episodes included repeated suicidal thoughts, previous depressive episodes, identification as

Risk factors for developing new depressive episodes in primary care

\begin{tabular}{lcc} 
Characteristic at baseline & Odds ratio & $95 \%$ Cl \\
Recognition as psychiatric case by GP & 1.5 & 1.1 to 2.2 \\
\hline Repeated suicidal thoughts ('crossed my mind') & 1.9 & 1.2 to 3.2 \\
\hline Previous depressive episodes & 1.7 & 1.2 to 2.6 \\
\hline Two or more chronic organ diseases & 1.4 & 0.9 to 2.1 \\
\hline High psychological distress & 2.2 & 1.1 to 4.3 \\
\hline ICD-10 current disorder & 4.8 & 2.1 to 10.5 \\
\hline Female gender & 1.1 & 0.8 to 1.6 \\
\hline
\end{tabular}

a psychiatric case by general practitioners, chronic organ diseases, poor general health and full or subthreshold ICD-10 disorder (table).

\section{Conclusions}

Sociodemographic factors were of less importance than psychological problems for predicting depressive episodes in primary care.

\section{COMMENTARY}

This is one of a number of reports due to emerge from WHO's recently completed study of the frequency, recognition, management and outcome of mental disorder in primary care. Spread over 14 countries, the study's size and sophistication are breath-taking. Screening tools and diagnostic instruments were reliable and valid; contact rates initially, and at one-year follow-up, were acceptable, and the results are reported in detail.

Findings from this report, which focuses on risk factors for new depressive episodes, are mostly predictable. People at greatest risk of developing ICD-10 depressive disorders over a 1-year period were those with some other mental disorder at baseline, a history of depression and repeated suicidal thoughts. Thus, mental disorders in the community are often co-morbid, chronic and relapsing.

Negative results are more notable here than positive ones. Women were at no greater risk of new-onset depression than men, despite their higher rate of depression in most prevalence studies. Age had no apparent bearing, despite the lower rates of depression in older people reported in other studies. The most likely explanation is that women and younger people remain unwell for longer periods once mental disorder strikes. Such subtleties will become clear only when the full results of this study are presented.

Large-scale studies like this serve a genuine sociopolitical purpose: politicians and managers are reminded that mental disorder is common, persistent and disabling. At the same time, broad-brush investigations might miss the subtle personal and communal influences that alter the risk of depression in particular communities.

Daniel O'Connor, MD, FRANZCP Professor of Psychiatry of Old Age

Monash University

Melbourne, Australia 